\title{
Hautveränderung nach Harnwegsinfekt
}

\section{Flammend rote Beine}

\section{Zunächst schien alles nach Plan zu laufen. Die rüstige 72-jährige Pati- entin erholte sich unter fünftägiger antibiotischer Therapie rasch von einem unkomplizierten Harnwegsin- fekt. Einige Tage später jedoch ent- wickelte sie plötzlich eine flammen- de Rötung an beiden Beinen. Ein Erysipel, eine Allergie? Oder etwas ganz anderes?}

— Die 72-jährige Patientin, bei der bisher keine ernsthaften gesundheitlichen Probleme bekannt waren, erkrankte an einem unkomplizierten Harnwegsinfekt. Unter einer fünftägigen Therapie mit der Kombination Trimethoprim/Sulfamethoxazol bildeten sich die Beschwerden relativ rasch zurück. Einige Tage später entwickelten sich fleckige Rötungen im Bereich beider Beine, die etwas erhaben erschienen und zu ausgedehnten Plaques mit flammender Röte konfluierten.

Fieber bestand nicht und die Entzündungsparameter waren nicht wesentlich erhöht. Da die Patientin angab, ihre Beine regelmäßig mit Franzbranntwein einzureiben, wurde neben einer Infektion i. S. eines Erysipels auch an ein allergisches Ekzem gedacht, wogegen allerdings der fehlende Juckreiz sprach.

\section{Genaue Inspektion der Haut}

Im proximalen Randbereich der flächigen Rötung fanden sich kleine, um-

\section{Fabula docet}

Bei einer plötzlich einsetzenden flammenden Rötung im Bereich beider Unterschenkel sollte immer an eine leukozytoklastische Vaskulitis gedacht werden, die durch Infektionen, Medikamente oder Autoimmunerkrankungen ausgelöst werden kann. Schon mittels klinischem Befund gelingt in den meisten Fällen die eindeutige Abgrenzung gegenüber einem Erysipel oder einem allergischen Kontaktekzem. schriebene, erhabene, fleckige Rötungen im Sinne einer Purpura. Dieser Befund sprach für das Vorliegen einer Vaskulitis, genauer gesagt einer kutanen leukozytoklastischen Vaskulitis. Unter Gabe von Kortison (beginnend mit $100 \mathrm{mg}$ in rasch ausschleichender Dosierung) bildete sich die Rötung innerhalb von zwei Wochen fast vollständig zurück.

\section{Vaskulitis der kleinen Gefäße}

Die leukozytoklastische Vaskulitis ist eine Vaskulitis der kleinen Gefäße, der Kapillaren und vor allem der Venolen. Ausgelöst wird das Krankheitsbild durch im Blut zirkulierende Immunkomplexe, die sich an die Gefäßwand anlagern und hier das Komplementsystem aktivieren. Dadurch wird die Gefäßpermeabilität erhöht und es setzt eine granulozytäre Chemotaxis ein. Die angelockten, vorwiegend eosinophilen und neutrophilen Granulozyten entleeren ihre Lysosomen und verstärken somit den Entzündungsprozess mit konsekutiver Gewebsschädigung.

\section{Vielerlei Auslöser}

Das Spektrum der auslösenden Ursachen einer solchen Vaskulitis reicht von Autoimmunerkrankungen wie rheumatoider Arthritis bzw. Lupus erythematodes über bakterielle und virale Infektionen bis hin zu Medikamenten. Die häufigsten auslösenden Substanzen sind Penicilline, Sulfonamide, Thiazide, Allopurinol, Phenytoin und nicht steroidale Antirheumatika. Auch maligne Grunderkrankungen können in Form eines paraneoplastischen Syndroms zu dieser Hautkomplikation führen. Bei fehlender Ursache spricht man von einer idiopathischen Vaskulitis.

\section{Typischer Befund: Tastbare Purpura}

Die charakteristische Hautveränderung bei der leukozytoklastischen Vaskulitis ist die tastbare Purpura. Dabei handelt es sich um erhabene, erythematöse,

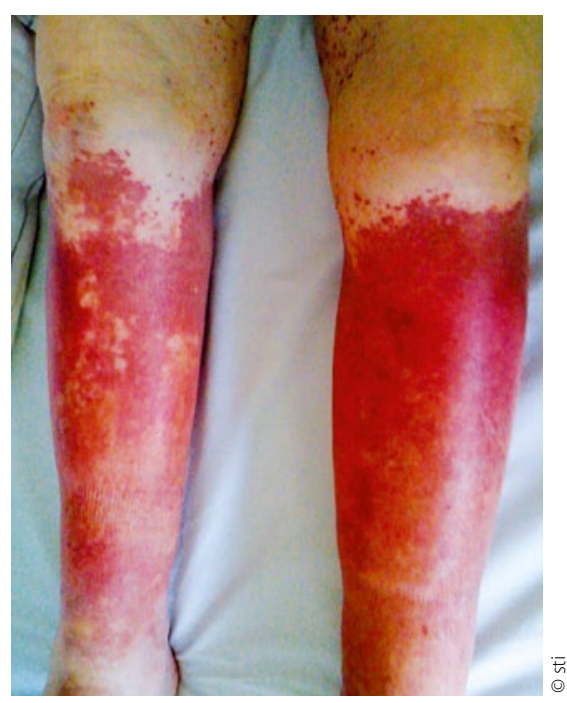

Ausgedehnte flächige Rötung an beiden Unterschenkeln mit Purpura.

nicht wegdrückbare Papeln, die zu größeren Plaques konfluieren können. Darüber hinaus können Nekrosen und Ulzerationen auftreten.

Besonders betroffen sind die durch hydrostatische Hämostase prädisponierten unteren Extremitäten sowie die Glutealregion bei bettlägerigen Patienten. Dagegen sind Gesicht, Handteller und Schleimhäute nur selten betroffen. Die Hautveränderungen können über einige Wochen persistieren und dann mit vorübergehender Hyperpigmentation, aber auch unter Narbenbildung abheilen.

In der Hautbiopsie finden sich typischerweise entzündliche Infiltrate in der Dermis mit Granulozyten, aber auch Lymphozyten, darüber hinaus auch $\mathrm{Ne}$ krosen an den Blutgefäßen mit sekundärer Fibrinablagerung und Blutungen in das perivaskuläre Gewebe. Durch Spezialfärbungen können evtl. auch Immunkomplexe an den Gefäßwänden nachgewiesen werden.

\section{Symptomatische Therapie}

Soweit eine krankheitsauslösende Ursache vorliegt, sollte diese beseitigt werden. Dazu gehört z. B. das Absetzen eines Medikaments. Ansonsten empfiehlt sich therapeutisch bei leichteren Formen ein Antihistaminikum oder Colchicin, bei schwereren Verlaufsformen sind systemische Glukokortikoide, evtl. auch Azathioprin oder Cyclophosphamid indiziert.

- Dr. med. Peter Stiefelhagen, Hachenburg 